\title{
Immunity and COVID-19 vaccines in pregnancy - precautions and risks
}

\author{
Anca A. SIMIONESCU ${ }^{1,2}$, Gabriel Cristian BEJAN ${ }^{3}$, Ana-Maria ADAM ${ }^{4}$, \\ Ana Maria Alexandra STANESCU ${ }^{3}$ \\ ${ }^{1}$ Department of Obstetrics and Gynecology, \\ "Carol Davila" University of Medicine and Pharmacy, Bucharest, Romania \\ ${ }^{2}$ Filantropia Clinical Hospital, Bucharest, Romania \\ ${ }^{3}$ Department of Family Medicine, "Carol Davila" University of Medicine and Pharmacy, Bucharest, Romania \\ ${ }^{4}$ Department of Surgery, "Dunarea de Jos" University of Medicine and Pharmacy, Galati, Romania
}

\begin{abstract}
Symptomatic coronavirus disease (COVID-19) during pregnancy represent a high-risk situation for maternal complications, including respiratory distress, pneumonia and even death. Pregnancy represents a unique adaptative immunological and hormonal state at the inflammatory response. At this time, global immunization is a promising way to stop this pandemic. Since early December 2020, more than 150 vaccine candidates in various stages of development and research are communicated. In Romania, there are four vaccines authorized by the European Medicines Agency (EMA): Pfizer-BioNTech, Moderna, OxfordAstraZeneca and Johnson \& Johnson. However, many people are reluctant to take the COVID-19 vaccine, including false beliefs about this use during pregnancy. Herein, we present the current state of knowledge about immunity and COVID-19 vaccines during pregnancy based on reported cases from medical literature. Most of them are reported from healthcare pregnant women in COVID-19 facilities or in women in the first trimester of pregnancy who did not know they were pregnant at the time of vaccination. The Eudravigilance from European Medicines Agency (EMA) constantly monitors any side effects after a vaccine. COVID-19 vaccination during pregnancy is proving to be as safe and effective as in the general population. Because COVID-19 vaccines have been observed in association with a higher rate of miscarriage in early pregnancy, it should be used with caution in the first trimester. Vaccination before planning a pregnancy in order to gain immunity at the time of conception is recommended.
\end{abstract}

Keywords: COVID-19 vaccines, pregnancy, mRNA vaccines

\section{INTRODUCTION}

Symptomatic coronavirus disease (COVID-19) during pregnancy represent a high-risk situation for maternal complications, including respiratory distress, pneumonia, risk of cesarean section, intubation and even death. Vaccines should end the pandemics and prevent the occurrence of a new one. COVID-19 vaccines are available, and their safety was proved by clinical trials since $(1,2)$. Vaccination has suggested high efficacy for preventing severe COVID-19 disease in the general population. Because pregnant women were not included in clinical trials of COVID-19 vaccines, many questions about safety for mother and fetus remain to be answered.

By early April 2021, more than 160 million people worldwide were infected or reinfected with SARS-CoV-2, and 2 million severe COVID-19 and 3.3 million death 
globally were reported (3). Severe acute respiratory syndrome coronavirus 2 (SARS-CoV-2 virus) was the leading cause of the aggravation of comorbidities and death (4).

Herein, we present the current state of knowledge about immunity and COVID-19 vaccines during pregnancy based on reported cases from medical literature. Most of them are reported from healthcare pregnant women in COVID-19 facilities or in women in the first trimester of pregnancy who did not know they were pregnant at the time of vaccination.

\section{PREGNANCY AND COVID-19}

In a systematic review of 2,567 pregnancies from 17 studies published since May $7^{\text {th }}$ 2020, Khalil et al. have calculated that one in five infected pregnant women will experience no symptoms, but symptomatic patients are at high risk of morbidity and mortality. 73.9\% were diagnosed in the third trimester. Fever was founded in $63,3 \%$ of cases, cough in $71.4 \%$ dyspnoea in $34.4 \%$ and $~ 15-20 \%$ presented non-specific complaints of myalgia, fatigue, headache or anosmia. Lymphopenia and raised inflammatory markers of C-reactive protein or procalcitonin were diagnosed in $34.2 \%$ of cases. Few women had evidence of co-infection. Forty-three maternal deaths were reported from the 2,567 reported cases, and $7 \%$ of cases were admitted in intensive care units and $3,4 \%$ with mechanical ventilation. Preterm birth, primarily iatrogenic, due to the worsening mother evolution was founded, in around one-fifth of cases, with half of the births by caesarean section. Perinatal deaths occurred in less than $1 \%$, twelve stillbirths and four neonatal deaths were registered. The rate of neonatal SARS-CoV-2 positivity was $1-2 \%$ (5).

A multicentric multinational prospective randomised study from Villar et al. (The INTERCOVID Multinational Cohort Study) of 706 pregnant women with COVID-19 diagnosis has reported, with almost $93 \%$ laboratory/radiological confirmation. $44.0 \%$ of women with RT PCR positive test were asymptomatic. Almost half of women in the group with COVID-19 diagnosis had overweight early in pregnancy compared with $40.2 \%$ of the group without COVID-19 diagnosis. Women with a COVID-19 diagnosis had higher rates of pregnancy-induced hypertension (RR, 1.46; $95 \% \mathrm{Cl}$, 1.052.02), preeclampsia/eclampsia (RR, 1.76; $95 \% \mathrm{Cl}$, 1.27-2.43), and infections requiring antibiotics (RR, 3.38; 95\% Cl, 1.63-7.01). Also, an association with a greater risk of admission to ICU/high-dependency unit (RR, 5.04; 95\% Cl, 3.13-8.10) and referral to a higher level of care (RR, 6.07; $95 \% \mathrm{Cl}, 1.23-30.01$ ) was reported in the group of women with COVID -19 diagnosis. Among all ICU admissions, women with COVID-19 diagnosis stayed $3.73(95 \% \mathrm{Cl}, 2.37-5.86)$ days longer than women without COVID-19 diagnosis Eleven women (1.6\%) with COVID-19 diagnosis died (maternal mortality ratio, 159/10 000 births) from severe preeclampsia, respiratory failure and pulmonary embolism. More, two women developed fever, cough, and breathlessness within seven days of an uneventful delivery and died shortly after, despite ICU care. One in seven preterm birth, primarily iatrogenic, due to the worsening mother evolution due to comorbidities and SARS-CoV-2 infection was founded. The increase in prematurity is explained by the fact that women with COVID-19 diagnosis delivered earlier, mostly by cesarean section than those without COVID-19 diagnosis, after approximately 30 weeks' gestation (6).

\section{MATERNALADAPTABILITY, IMMUNITY AND TOLERANCE TO THE ALLOGENIC FETUS}

Pregnancy represents a unique adaptative immunological and hormonal state when the adaptive/inflammatory immunity is reduced (7-9).

Pregnant women represent a high-risk population for infectious and respiratory diseases due to a shift from cellular to humoral immunity (10). Physiological respiratory changes during pregnancy, such as reduced functional residual respiratory volumes, diaphragm elevation, hyperventilation, lead to increased susceptibility to viral infections and severe pneumonia (11).

The maternal immune system has to tolerate the semiallogeneic fetus with paternal antigens. Maternofetal immune tolerance is essential to maintain pregnancy and to fight any external environmental viral threats. HLA expression pattern which include HLA-E, $-F,-G$, and $-C$ is essential in maternal tolerance to the semiallogeneic fetus and fetal development. Different immunological mechanisms combined with different ratios among various populations of immune cells in the maternal-fetal interface may occur to modulate pregnancy-related pathologies, including gestational diabetes, preeclampsia, and metabolic syndrome $(8,9,12)$. Maternal immunological states adapt with the growth and development of semiallogenic fetus at a different phase of development: from a pro-inflammatory state in the first trimester to an anti-inflammatory state in the second and third trimester of pregnancy (13). Based on the knowledge that pregnant women in their first and third trimester are at the pro-inflammatory state, the cytokine-storm induced by SARS-CoV-2 may induce a more severe inflammatory state in these women (13).

The increase in pro-inflammatory cytokines at the beginning of the cytokine storm leads to viral sepsis (14).

An inflammatory response may induce lung injury. Moreover, some studies have shown that a neutrophil 
increase and a lymphocyte drop appear to be related to disease severity and even death, suggesting that lymphopenia may play a significant role in the pathogenesis of severity of COVID-19.

Pregnant women require frequent visits to the general practitioner and the maternity for prenatal care visits, ultrasound or laboratory tests. Many infected asymptomatic women have children who go to kindergarten and nursery, so there is a high risk of spreading the virus. Moreover, healthcare and frontline pregnant women are at higher risk for SARS-CoV-2 infections.

\section{COVID-19 VACCINATION DURING PREGNANCY}

Given all of the above, it seems common sense that pregnant women must be vaccinated as a high-risk population for the occurrence of complicated forms of COVID-19.

Since the COVID -19 vaccination introduction, taking into account more benefits than risks, the Romanian Society of Obstetrics and Gynecology (SOGR), according to the model of many authorized authority (Centers for Disease Control and Prevention - CDC, American College of Obstetricians and Gynecologists, American Academy of Pediatrics etc.) have issued guidance indicating that Covid-19 vaccines should not be withheld from pregnant persons. SOGR have argued during seminars and webinars about the safety and efficacies, and formulate recommendations on maternal vaccination against COVID-19 (15-18).

Despite controversies regarding fetal effects and the enrolment in trials vaccines of pregnant women, since 2013, the National Institutes of Health's National Institute of Allergy and Infectious Diseases organized a series of conferences and established an expert panel that developed guidelines and provide general principles to guide clinical investigators on the assessment of the safety of vaccines in clinical trials in pregnant population persons (19).

SARS-CoV-2 contains four structural proteins, including spike (S), envelope (E), membrane (M), and nucleocapsid (N). Different cell surface receptors recognize $S$ proteins and ACE2 receptor bind with the viral $S$ protein and dictate viral particle enter the host. As of January 2020, the complete genome sequence analysis of SARS-CoV-2 was published (20). The genome of SARS-CoV-2 is comprised of a single-stranded positive-sense RNA (21). SARS-CoV-2 have the largest RNA viral genome, ranging from 26 to $32 \mathrm{~kb}$ in length DNA vaccines anti SARS-CoV-2 have been developed expressing $\mathrm{S}, \mathrm{M}$, and $\mathrm{N}$ proteins, protective humoral and cellular immune responses in mice, macaques, and camels (22).

As of April 2021, the global COVID-19 vaccine research and development landscape include 115 vac- cine candidates in various authorization, development and research (23). They are based on different technology: nucleic acid (DNA and RNA), virus-like particle, peptide, viral vector (replicating and non-replicating), recombinant protein, live attenuated virus and inactivated virus approaches. Fourteen vaccines are authorized by at least one national regulatory authority for public use: two RNA vaccines (Pfizer-BioNTech and Moderna), five conventional inactivated vaccines (BBIBP-CorV, CoronaVac, Covaxin, WIBP-CorV and CoviVac), five viral vector vaccines (Sputnik Light, Sputnik V, Oxford-AstraZeneca, Convidecia, and Johnson \& Johnson), and two protein S subunit vaccines (EpiVacCorona and RBD-Dimer). Thirty-five trials about COVID-19 and vaccines were found on the EU Clinical Trials Register (24).

U.S. Food and Drug Administration (FDA) and the European Medicines Agency (EMA) have been submitted an Emergency Use Authorization (EUA) for the two mRNA-based vaccines.

In Romania, there are four vaccines authorized by the EMA: Pfizer-BioNTech, Moderna, Oxford-AstraZeneca and Johnson \& Johnson. Pfizer-BioNTech vaccine needs to be kept minus 70 degrees Celsius, and Moderna needs to be frozen at minus 20 Celsius for up to 30 days before administration.

Moderna's vaccine is administered as two $100 \mathrm{mi}-$ crogram doses given 28 days apart. Pfizer's vaccine is administered as two 30-microgram doses given 21 days apart. Oxford-AstraZeneca vaccine is administered as two separate doses of $0.5 \mathrm{ml}$ given 4 and 12 weeks ( 28 to 84 days) after the first dose. Johnson \& Johnson vaccine is administered in a single dose of $0.5 \mathrm{ml}$.

Because pregnant women were not included in clinical trials of COVID-19 vaccines, available data are reported primarily on healthcare pregnant women from COVID-19 facilities or in women who did not know they were pregnant at the time of vaccination because the pregnancy status was not checked before.

Significant animal phase 1 and 2 studies on COVID-19 vaccination showed no adverse effects on fetal or embryonic development or interference in female reproduction capacity (25).

A very large report about mRNA COVID-19 vaccine safety on the outcomes of 827 pregnancy registered in the v-safe pregnancy registry, and the Vaccine Adverse Event Reporting System (VAERS) has reported 86.1\% live birth, $0.1 \%$ stillbirth and $12.6 \%$ spontaneous abortion. Receipt of the first dose of vaccine (Moderna or Pfizer-BioNTech) was reported by $2.3 \%$ participants during the periconception period, by $28.6 \%$ in the first trimester of pregnancy, by $43.3 \%$ in the second trimester, and by $25.7 \%$ in the third trimester. $91.9 \%$ participants received a vaccine in the first trimester, and $99.2 \%$ received a vaccine in the second trimester. The 
majority of abortion occurred in the first trimester of pregnancy, before 13 weeks of pregnancy, but cannot demonstrate a relationship between vaccination and miscarriage. $86.1 \%$ of patients gave birth. Newborn antibodies to SARS-CoV- 2 was detected in cord blood after third-trimester maternal vaccination. This may suggest that transplacental transfer of IgG SARS-CoV-2 antibodies after maternal Covid-19 vaccination occur in the third trimester of pregnancy. Maternal vaccination near term might provide some level of protection to the neonate (26).

Despite a small number of cases, other reports have to outline newborn antibodies to SARS-CoV-2 detected in cord blood after the first vaccine dose received in the third trimester, begging the question on antibody transfer and the level of possible neonatal protection relative to the mother's timing of vaccination.

An analysis about general consideration for counselling pregnant women has shown that the reported side-effects after mRNA vaccines were similar to side effects in the general population: muscle pain, fever, fatigue, headache, chills. Fever $\left(38^{\circ} \mathrm{C}\right.$ or higher) occurred in $3.7 \%$ of participants with a positive pregnancy test at the time of vaccination after dose 1 and $15.8 \%$ after dose 2 of the Pfizer-BioNTech mRNA. Antipiretics are authorized during pregnancy. Fever during pregnancy may be associated with maternal (infections), obstetrical (miscarriage, premature birth) and/ or fetal (fetal demise, birth defects) adverse outcomes (27). Given the vaccine reactogenicity, including immune fever $\left(38^{\circ} \mathrm{C}\right.$ or higher), vaccination in the first trimester could increase the risk of the neural tube and other congenital defects (28). The risk of complications to the pregnant person based on maternal comorbidities need to be considered: such as diabetes, obesity, hypertension or heart disease, which could cause an increased risk for severe COVID-19 complications (29).

The AstraZeneca vaccine was associated with a high risk of thromboses after few reported cases of this severe complication and death. Pregnant women have a higher risk of thrombosis and thromboembolism. So it is advisable to use mRNA vaccines during pregnancy.

\section{CONCLUSIONS}

Pregnant women are recommended to get a COVID-19 vaccination using mRNA vaccines (BioNTech/ Pfizer and Moderna vaccines). COVID-19 vaccination during pregnancy is considered to be safe and effective. But many questions remain for future research. Two of the important ones are the optimal time to get vaccinated - in the third-trimester maybe or secondly, about infants immunity for SARS-CoV-2 infection. Due to the high rate of early abortion reported in the SARS-CoV-2 population, prepregnancy vaccination may be useful.

Conflict of interest: none declared Financial support: none declared

\section{REFERENCES}

1. Walsh EE, Frenck RW Jr, Falsey AR, Kitchin N, Absalon J, Gurtman A, Lockhart S, Neuzil K, et al.; C4591001 Clinical Trial Group. Safety and Efficacy of the BNT162b2 mRNA Covid-19 Vaccine. N Engl J Med. 2020 Dec 31;383(27):2603-2615.

2. WHO Coronavirus disease 2019 (COVID 19).Available at: https://www.who coronavirus disease. Accessed on May 2021.

3. Horobet A, Simionescu AA, Dumitrescu DG, Belascu L. Europe's War against COVID-19: A Map of Countries' Disease Vulnerability Using Mortality Indicators. Int J Environ Res Public Health. 2020 Sep 9;17(18):6565.

4. Khalil A, Kalafat E, Benlioglu C, O'Brien P, Morris E, Draycott T, Thangaratinam S, Le Doare K, Heath P, Ladhani S, von Dadelszen P, Magee LA. SARS-CoV-2 infection in pregnancy: A systematic review and meta-analysis of clinical features and pregnancy outcomes. EClinicalMedicine. 2020 Aug;25:100446.

5. Villar J, Ariff S, Gunier RB, Thiruvengadam R, Rauch S, Kholin A, Roggero P, Prefumo F, et al. Maternal and Neonatal Morbidity and Mortality Among Pregnant Women With and Without COVID-19 Infection: The
INTERCOVID Multinational Cohort Study. JAMA Pediatr. 2021 Apr 22:e211050.

6. Kraus TA, Engel SM, Sperling RS, Kellerman L, Lo Y, Wallenstein S, Escribese MM, Garrido JL, Singh T, Loubeau M, Moran TM. Characterizing the pregnancy immune phenotype: results of the viral immunity and pregnancy (VIP) study. J Clin Immunol. 2012 Apr;32(2):300-11.

7. Martínez-Varea A, Pellicer B, Perales-Marín A, Pellicer A. Relationship between maternal immunological response during pregnancy and onset of preeclampsia. J Immunol Res. 2014;2014:210241.

8. Siddiqui S, Waghdhare S, Jha S, Dubey S. Role of immunological markers in gestational diabetes mellitus-a brief review. Diabetes Metab Syndr. 2019 SepOct;13(5):2983-2985.

9. Kourtis AP, Read JS, Jamieson DJ. Pregnancy and infection. N Engl J Med. 2014 Jun 5;370(23):2211-8.

10. Wu C, Yang W, Wu X, Zhang T, Zhao Y, Ren W, Xia J. Clinical Manifestation and Laboratory Characteristics of SARS-CoV-2 Infection in Pregnant Women. Virol Sin. 2020 Jun;35(3):305-310.
11. Laresgoiti-Servitje E, Gómez-López N, Olson DM.An immunological insight into the origins of pre-eclampsia. Hum Reprod Update. 2010 Sep-Oct; 16(5):510-24.

12. Mor $G$, Aldo $P$, Alvero AB. The unique immunological and microbial aspects of pregnancy. Nat Rev Immunol. 2017 Aug;17(8):469-482.

13. Prompetchara E, Ketloy C, Palaga T. Immune responses in COVID-19 and potential vaccines: Lessons learned from SARS and MERS epidemic. Asian Pac J Allergy Immunol. 2020 Mar;38(1):1-9.

14. https://sogr.ro/wp-content/uploads/2021/01/ POZITIA-SOCIETATII-DE-OBSTETRICA-SIGINECOLOGIE-DIN-ROMANIA-FATA-DEVACCINAREA-COVID-19-A-FEMEIIGRAVIDE-SI-CARE-ALAPTEAZA.pdf THE POINT-OF-VIEW-OF-THE-OBSTETRICSAND-GYNECOLOGY-SOCIETY-OFROMANIA-THE-FACE-OF-COVIDVACCINATION-19-OF-PREGNANT-ANDBREASTFEEDING-WOMEN.pdf.

15. Centers for Disease Control and Prevention. COVID-19 vaccines: interim clinical considerations for use of COVID-19 vaccines currently authorized in the United States. 2021. Available at: https://www.cdc. 
gov/vaccines/covid-19/info-by-product/ clinical-considerations.html.

16. American College of Obstetricians and Gynecologists. Vaccinating pregnant and lactating patients against COVID-19: practice advisory. December 2020. Available at: https://www.acog.org/clinical/clinicalguidance/practice-advisory/articles/2020/12/ vaccinating-pregnant-and-lactating-patientsagainst-covid-19.

17. American Academy of Pediatrics. Interim guidance for COVID-19 vaccination in children and adolescents 2021. Available at: https://services.aap.org/en/pages/2019novel-coronavirus-covid-19-infections/ clinical-guidance/interim-guidance-for-covid19-vaccination-in-children-and-adolescents/.

18. Munoz FM, Sheffield JS, Beigi RH, Read JS, Swamy GK, Jevaji I, et al. Research on vaccines during pregnancy: protocol design and assessment of safety. Vaccine. 2013 Sep 13;31(40):4274-9.

19. Lu R, Zhao X, Li J, Niu P, Yang B, Wu H, Wang W, et al. Genomic characterisation and epidemiology of 2019 novel coronavirus: implications for virus origins and receptor binding. Lancet. 2020 Feb

22;395(10224):565-574.

20. Naqvi AAT, Fatima $K$, Mohammad T, Fatima U, Singh IK, Singh A, Atif SM, Hariprasad G, Hasan GM, Hassan MI. Insights into SARS-CoV-2 genome, structure, evolution, pathogenesis and therapies: Structural genomics approach. Biochim Biophys Acta Mol Basis Dis. 2020 Oct 1;1866(10):165878.

21. Muthumani K, Falzarano D, Reuschel EL, Tingey C, Flingai S, Villarreal DO, et al. A synthetic consensus anti-spike protein DNA vaccine induces protective immunity against Middle East respiratory syndrome coronavirus in nonhuman primates. $\mathrm{Sci}$ Transl Med. 2015 Aug 19;7(301):301ra132.

22. Thanh Le T, Andreadakis Z, Kumar A, et al. The COVID-19 vaccine development landscape. Nat Rev Drug Discov. 2020 May;19(5):305-306.

23. EU - https://www.clinicaltrialsregister.eu/ ctr-search/search?query=covid19+and+vaccines accesed on 01 May 2020.

24. U.S. Food and Drug Administration. Vaccines and Related Biological Products Advisory Committee meeting-December
17, 2020_FDA briefing documentModerna COVID-19 vaccine. Accessed on December 15, 2021.

25. Shimabukuro TT, Kim SY, Myers TR, Moro $\mathrm{PL}$, Oduyebo T, Panagiotakopoulos $\mathrm{L}$, Marquez PL, et al.; CDC v-safe COVID-19 Pregnancy Registry Team. Preliminary Findings of mRNA Covid-19 Vaccine Safety in Pregnant Persons. N Engl J Med. 2021 Jun 17;384(24):2273-2282.

26. Rasmussen SA, Kelley CF, Horton JP, Jamieson DJ. Coronavirus Disease 2019 (COVID-19) Vaccines and Pregnancy: What Obstetricians Need to Know. Obstet Gynecol. 2021 Mar 1;137(3):408-414.

27. Egloff C, Sibiude J, Couffignal C, Mandelbrot $\mathrm{L}$, Picone O. Causes and consequences of fever during pregnancy: A retrospective study in a gynaecological emergency department. J Gynecol Obstet Hum Reprod. 2020 Nov;49(9):101899.

28. Graham JM Jr. Update on the gestational effects of maternal hyperthermia. Birth Defects Res. 2020 Jul 15;112(12):943-952. 